\title{
Light-mediated planar polarization of cone photoreceptor cilia contributes to visual acuity in mammals
}

Michael Housset ${ }^{1 *}$, Dominic Filion ${ }^{2}$, Nelson Cortes $^{3}$, Hojatollah Vali ${ }^{4}$, Craig Mandato ${ }^{5}$, Christian Casanova ${ }^{3}$, Michel Cayouette ${ }^{1,5,6,{ }^{*}}$

1 Cellular Neurobiology Research Unit, Institut de Recherches Cliniques de Montréal (IRCM), Montreal, QC, H2W 1 R7

2 Microscopy Core Facility, Institut de Recherches Cliniques de Montréal (IRCM), Montreal, QC, H2W 1R7

3 School of Optometry, Université de Montréal, CP 6128 succursale centre-ville, Montreal, QC H3C 3J7, Canada.

4 Facility for Electron Microscopy Research, McGill University, Montreal, QC, H3A $0 \mathrm{C} 7$

5 Department of Anatomy and Cell Biology, McGill University, Montreal, QC, H3A 0C7

6 Department of Medicine, Université de Montréal, Montreal, QC, H3T 1J4

*michael.housset@ircm.qc.ca; michel.cayouette@ircm.qc.ca 


\begin{abstract}
Planar cell polarity (PCP) is essential to optimize information processing and functional response in many tissues. While the fly eye is a classic example of PCP, it remains unknown whether PCP exists in the mammalian retina and whether it plays a part in vision. Here we used $3 D$ reconstructions of the mouse retina to show that the basal body of cone photoreceptor cilia, but not rods, is systematically located on the side of the cell facing the centre of the retina. We further show that light is required during a critical window of development to establish cone PCP, and that both cone transducin and the G-protein signaling modulator protein 2 are required to mediate this effect. Importantly, we report that disruption of cone PCP impairs visual acuity. This work uncovers a non-canonical PCP pathway, mediated by light, and identifies cone PCP as a feature supporting mammalian vision.
\end{abstract}




\section{INTRODUCTION}

The range of information an organism can discriminate in its visual field is highly dependent on the diversity, quantity and spatial arrangement of light-sensing photoreceptor cells in the retina. The vertebrate retina contains rod and cone photoreceptors to detect dim or bright light, respectively, with cones additionally sensitive to specific wavelengths. In both rods and cones, the photosensitive cilia, or outer segment (OS), develops from the connecting cilium, a microtubule-based structure anchored by a basal body to the apical surface of the inner segment (IS). While the OS is exclusively devoted to detecting light, the IS houses organelles, such that proteins essential for phototransduction are translated into the IS and transported to the OS along the connecting cilium (1).

In addition to apicobasal polarity, epithelial cells often display coordinated alignment of polarized cell-intrinsic features across the tissue plane, a process known as planar cell polarity (PCP). Remarkable examples of PCP are found in Drosophila, such as the uniform alignment of sensory bristles along the body wall or the precise alignment of ommatidia containing different photoreceptor cell types arranged in a stereotyped fashion across the eye (2-4). In the latter, PCP is essential for tissue patterning, but may also optimize visual perception by facilitating synaptic convergence of identically oriented photoreceptors to the brain, a wiring process known as neuronal superposition $(5,6)$. In the mammalian retina, while the mechanisms and role of photoreceptor apicobasal polarity in vision have been extensively studied $(7,8)$, it remains unknown whether photoreceptors display PCP and, if so, whether this might have a role in vision.

A classic model of PCP in vertebrates is the mechanosensory hair cells of the inner ear, which display a V-shaped bundle of mechanosensitive stereocilia oriented in a coordinated manner across the tissue to optimize sound detection $(9,10)$. Critical to establishing this PCP is the migration of the primary kinocilium from the centre of the apical surface to the lateral edge. We and others have reported that relocalization of the kinocilium requires the adaptor protein Inscuteable, the G-protein signaling modulator 2 (Gpsm2, also known as LGN) and the heterotrimeric G protein Gai (11, 12 ), a protein complex well-known for its role in regulating mitotic spindle orientation (13-16). Analogous to the hair cell kinocilium, the connecting cilium of photoreceptors is thought to be anchored eccentrically by the basal body at the edge of the IS, thereby constituting a possible polarized cell-intrinsic feature in photoreceptors. However, whether connecting cilia are coordinately oriented to establish PCP in the mammalian retina is unknown. An interesting parallel can be made between the G-protein signalling regulating kinocilium placement in hair cells and the phototransduction cascade, which also relies on a heterotrimeric G-protein. When photons hit the OS disk membranes, the 11-cis retinal chromophore isomerizes to all-trans, which serves as the ligand for the G-protein coupled receptor opsin. This initiates a biochemical cascade that enables the association of opsin with 
transducin (Gat), a heterotrimeric G-protein related to the Gai family, which in turn activates the cyclic GMP-phosphodiesterase that controls cGMP-gated ion channels at the photoreceptor plasma membrane $(8,17,18)$. Of note, Gpsm2 is the only guanine dissociation inhibitor (GDI) protein expressed in mouse photoreceptors, and it interacts with the alpha subunit of rod transducin (Gat1) in a GDP-bound state (19, 20). Based on these observations, we hypothesized that light might trigger a Gprotein signalling cascade modulated by Gpsm2, which would lead to the asymmetric positioning of connecting cilia and establishment of PCP in photoreceptors. We further postulate that this organization may be important to optimize visual detection. Here we used high-resolution 3D reconstructions of the retina, dark-rearing, genetics, and visual behaviour assays to test this hypothesis in the mouse retina. 


\section{RESULTS}

\section{Cone photoreceptors exhibit planar cell polarity in the mammalian retina}

Classically, the connecting cilium is presented as eccentrically anchored at the surface of the IS, but whether this is a general feature in all photoreceptors remains unknown. To begin to tackle this fundamental question, we first investigated the ultrastructure of the mouse retina in 3D using Focused lon Beam Scanning Electron Microscopy (FIBSEM) to obtain hundreds of serial images in the region of the connecting cilia. Cross-section and en-face milling were collected in the dorsal region of two independent adult retinas (Fig. 1a, b). Computational segmentation of the different sub-compartments of photoreceptors (IS, OS, basal body and connecting cilium) was carried out for each individual rod and cone identified in the volume of acquisition (Fig. 1c, FigS1a, Movie S1). By analyzing the relative distance of the basal body to the center of the IS at the surface of each photoreceptor, we discovered that the basal body is located systematically next to the edge of the IS in both rods and cones (Fig. 1a', a", b', b", d), confirming the general assumption that the connecting cilium is eccentrically anchored at the surface of photoreceptors.

We next investigated whether this polarized cell-intrinsic feature may be coordinated across the retina. To do this, we measured the planar orientation of basal bodies in individual rod and cone photoreceptors, relative to a centripetal reference axis, defined as the center-to-periphery axis of the retina. To help quantify the degree of planar polarity, the cumulative distribution of basal body orientations was fitted to a probability function in a polar referential (Fig. 1e, Fig. S1b), generating for each set of orientations a polarity score ( $\kappa$, kappa) and a preferred orientation $(\mu, \mathrm{mu})$, as previously described (21). The higher the kappa, the more concentrated around a single value the orientations are, with $2 / \mathrm{k}$ representing the $95 \%$ confidence interval in radians. We found that basal bodies are orientated randomly among the rod population, with kappa values below 0.4 in both acquisitions. In contrast, we found that basal bodies of cones are consistently located on the side of the IS that is closest to the optic nerve head (Fig. 1f, g, Fig. S1c, d), with polarity scores of 20 and 2.3 in the cross-sectional and en-face acquisitions, respectively.

Although robust, the coordinated orientation of basal bodies could only be studied for a small number of cones by FIBSEM, as the mouse retina is rod-dominated. To determine whether cones exhibit PCP in all regions of the retina, we used a method that enables a higher throughput of measurements by staining cone IS with peanut agglutinin lectin (PNA) and centrosomes with pericentrin (Pcnt). We then used confocal microscopy to acquire z-stacks from retinal flatmounts and measured centrosome eccentricities and orientations using the same method as for the FIBSEM experiments described above (Fig. 1h). We found that, independent of their location across the retina, cones consistently display eccentric centrosomes located on the side of the cell facing towards the optic nerve head (Fig. 1 i-k, Fig. S1e). 
Taken together, these results formally demonstrate cell-intrinsic polarity in all photoreceptors and uncover planar polarization of cones in the mammalian retina.
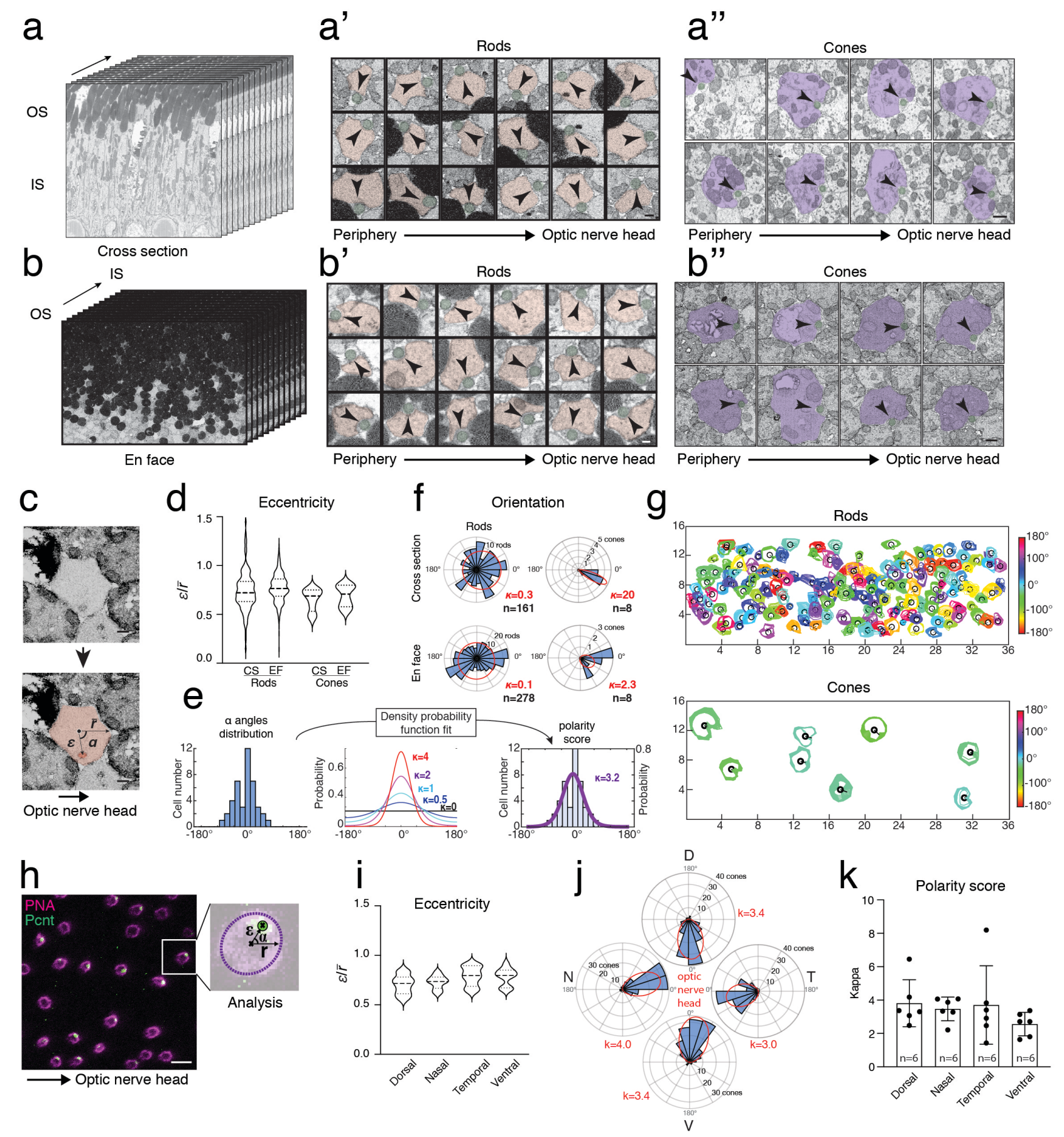

Figure 1. Cone planar cell polarity in the mouse retina

(a-b) Cross-section (a; 628 images, 25nm step) and en face (b; 914 images, 23nm step) FIBSEM acquisitions. High magnification views of different rods (a', b') and cones (a", b") in the connective cilium region. Orientation of the cilium (green) is indicated relative to the center of rod (red) and cone (purple) IS by an arrowhead. (c) Method for measurement of eccentricity and orientation of the basal body. Basal body (dark red) and IS (light red) are segmented and eccentricity of the basal body is measured by the ratio of the distance $\varepsilon$ 
between the IS and basal body and the mean radius $\bar{r}$ of cone IS. The orientation $a$ is defined by the angle between the IS-basal body axis and the center-to-periphery axis, using the optic nerve head as a reference point. (d) Violin plots of rod and cone basal body eccentricity. CS: cross-section ( $n=164$ rods; 8 cones), EF: en-face ( $n=280$ rods; 8 cones). (e) Basal body orientation analysis workflow: $\alpha$-angle distribution is fitted to density probability function in a polar referential, resulting in generation of a polarity score ( $\mathbf{k}$, kappa). See Experimental Procedures for details. (f) Polar histograms of basal body orientations (blue bins) and the associated density probability fit (red ellipse). (g) Top view of rod (top) and cone (bottom) IS membranes (coloured) and basal body (black circle) colour-coded for the $\alpha$ value obtained in cross-section acquisition. (h) Confocal acquisition of a mouse retina flatmount stained for peanut agglutinin lectin (PNA, magenta) and pericentrin (green). Centrosome eccentricity and orientation were measured using the same method as shown in c. (i) Violin plot of cone centrosome eccentricity in different regions of the adult mouse retina by confocal analysis ( $n=40$ cones from 3 different adult retinas). (j) Polar histograms of basal body orientations (blue bins) from different regions of the adult mouse retina: Dorsal (D); Nasal (N); Temporal (T); Ventral (V). (k) Histogram of cone polarity scores (kappa values) in different regions of the adult mouse retina. Graph shows mean \pm s.d. $n$ indicates biological replicates. Total number of cones analyzed were 537 dorsal, 664 nasal, 675 temporal, 628 ventral (see also Fig. S1e). One-way ANOVA $p=0.45$. Scale bars: $300 \mathrm{~nm}$ (a',b',c), 500nm (a", b"), $10 \mu \mathrm{m}(\mathrm{h})$.

\section{The establishment of cone PCP requires exposition to light during a critical period}

Cones are amongst the earliest-born cell types in the retina, with peak genesis around embryonic day 14.5 (E14.5). The OS, however, only starts to grow from the connecting cilium around postnatal day 8 (P8) (22). To identify when PCP emerges during cone morphogenesis, we measured centrosome orientations at different developmental stages using confocal acquisitions of immunostained retinal flatmounts (Fig. 2a). We found that centrosomes are distributed randomly at the surface of cone IS before P5, and shift to an eccentric position between P5 and P8, although they do not yet display a coordinated direction toward the optic nerve head ( $\leqslant \leq 0.5$, Fig. 2b, c, Fig. S2a). Between P12 and P14, centrosome orientations become coordinated, with kappa values increasing from $0.4 \pm 0.2$ at $\mathrm{P} 12$ to $1.8 \pm 0.9$ at P14, and stabilized past P14 (Fig. 2c, Fig. S2a).

Although gradients of morphogens are classically known to regulate PCP, our observation that cone PCP is established concomitantly to eyelid opening in mice (P12-14) prompted us to hypothesize that light may instead play a part in instructing cone PCP. To test this idea, we raised mice in constant darkness from E14.5 and studied cone PCP at P30. Remarkably, light deprivation prevented the emergence of cone PCP, without affecting the eccentric localization of centrosomes in cones (Fig. $2 \mathrm{~d}, \mathrm{~h}-\mathrm{i}$, Fig. S2b), indicating that a light-dependent mechanism is required to instruct cone PCP. To determine whether there might be a critical window for this lightdependent mechanism, we raised mice in normal light-dark cycles until P9, P12 or P16, and then switched them to constant darkness until P30 when we measured 
cone PCP. While cone centrosomes were randomly oriented in mice exposed to light-dark cycles until P9 ( $\mathrm{k}=0.6 \pm 0.1$, Fig. S2b) and P12 ( $\mathrm{k}=0.7 \pm 0.1$; Fig. $2 \mathrm{e}$, Fig. $\mathrm{S} 2 \mathrm{~b})$, they exhibited a marked polarization in mice exposed to light-dark cycles until P16 ( $k=1 \pm 0.1$; Fig. 2f, Fig. S2b), although not to the same extent as control mice that were never dark-reared $(\mathrm{k}=3.0 \pm 0.2)$. Together, these results show that light is required to mediate cone PCP during a critical time window between P12 and P16.

Finally, we wanted to determine whether cone PCP could be restored by switching mice that were dark-reared through the critical window to light-dark cycles. We darkreared mice from $E 14.5$ to $P 22$, and then switched them to light-dark cycles until P360. We found that cone centrosomes still exhibit random orientations under these conditions (Fig. 2g-h, Fig. S2b), showing that, beyond P22, exposure to light does not restore the loss of cone PCP mediated by previous dark-rearing. Conversely, cone PCP was unaffected by switching adult mice raised in light-dark cycles to constant darkness for a month (Fig. 2h, Fig. S2b), suggesting that light is not required to maintain cone $\mathrm{PCP}$ past the critical time window.

Altogether, these results show that basal bodies are randomly distributed within the IS of individual cone photoreceptors at birth, then shift eccentrically around P8, and become coordinately aligned on the side of the IS closer to the optic nerve between P12 and P14 in a light-dependent manner. 
a
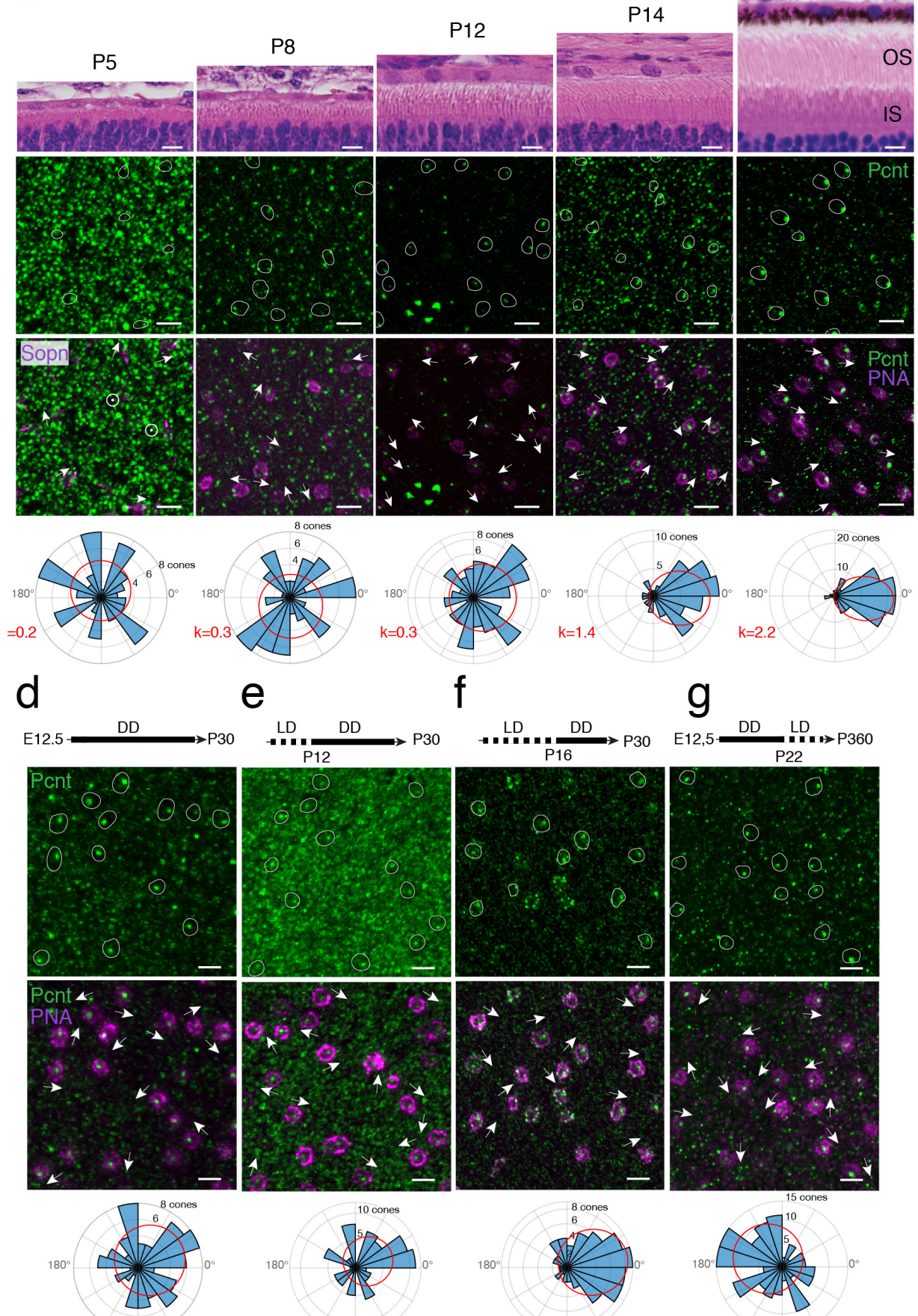

$k=0.4$

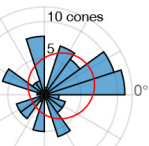

$\mathrm{k}=0.7$ f
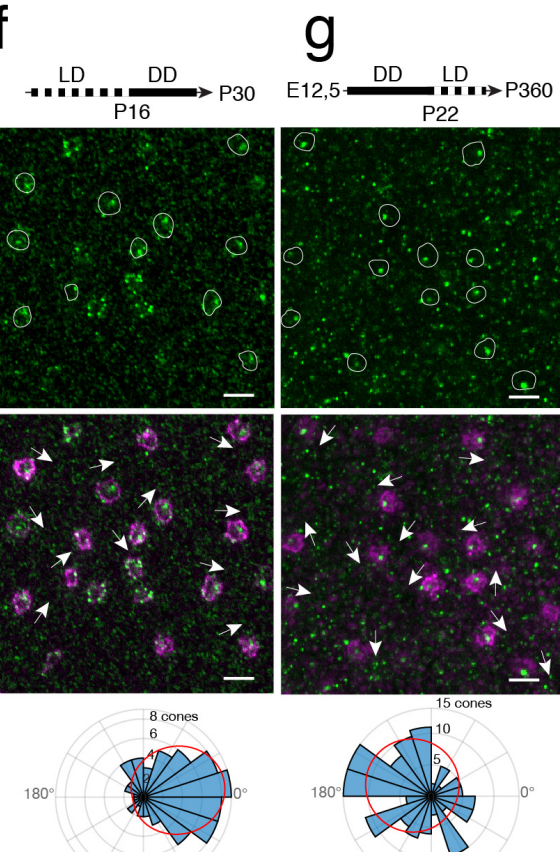

$k=1.0$

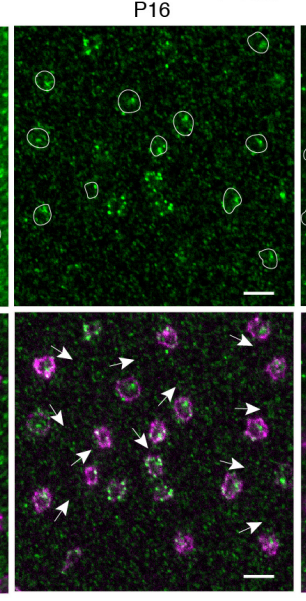

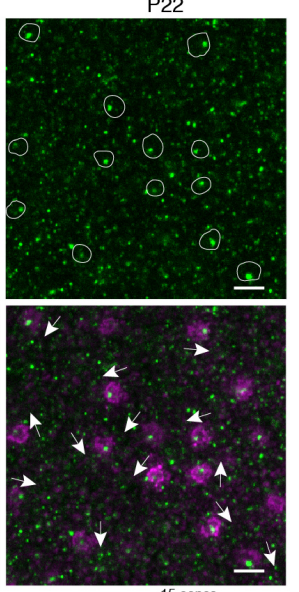

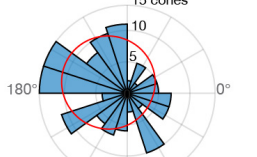

$\mathrm{k}=0.5$ b
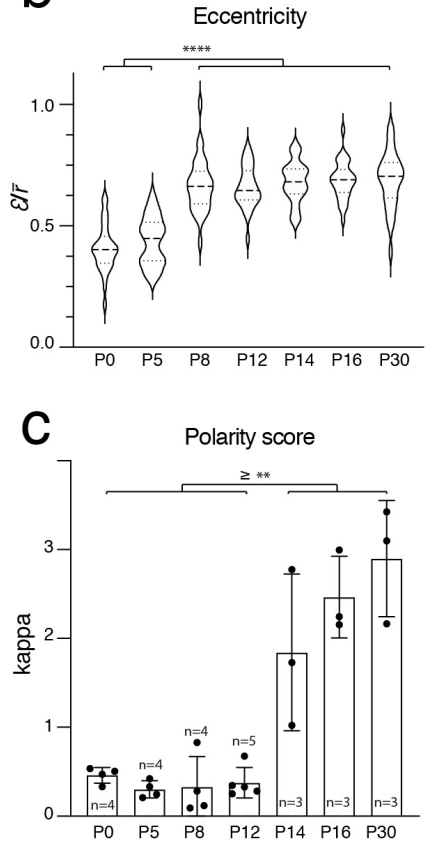

$\mathrm{h}$

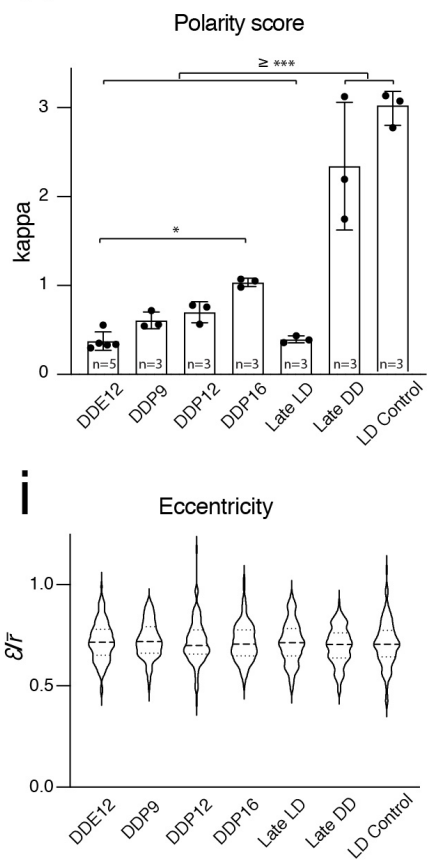

Figure 2. Critical period of cone PCP establishment

(a) Hematoxylin/eosin staining of mouse retinal sections at different stages, as indicated, and associated confocal acquisitions of flat-mounts of the contralateral retina stained for pericentrin (Pcnt, green), S-Opsin (Magenta, P5), or Peanut agglutinin (PNA, magenta). Cones are outlined by a white line and centrosome orientation is shown by an arrow in the bottom panels. Circles with a dot indicate a centrally-located centrosome (no orientation). For each stage, a representative rose plot of centrosome orientation ( $\alpha$-angles distribution) and associated kappa values is shown in the bottom row. (b) Violin plots of rod and cone 
basal body eccentricity ( $n=3$ animals per condition, 360 cells counted for each condition). One way ANOVA $p<0.0001$. Tukey multiple comparison test ${ }^{* * * *} p<0.0001$. (c) Histogram of cone polarity scores at different stages. Graph shows mean \pm s.d. $n$ indicates biological replicates. Total number of cones measured were: 339 at P0, 299 at P5, 355 at P8, 385 at $\mathrm{P} 12,1127$ at P14, 1389 at P16, and 1314 at P30. One-way Anova with Dunnett's multiple comparison test ${ }^{* *} p<0.01$. (d-g) Confocal acquisitions of retina flat-mounts stained for pericentrin (Pcnt, green) and Peanut agglutinin (PNA, magenta) from mice dark-reared (solid black line) between E12.5 and P30 (d), reared in light-dark cycles (dotted line) until P12, then dark-reared until P30 (e), reared in light-dark cycle until P16 and dark-reared until P30 (f), or dark-reared between E12.5 and P22 then reared in light-dark cycles until sacrifice at P360 (e). Representative examples of cones (outlined) with various centrosome orientation (arrows) are shown. For each stage, a representative rose plot of centrosome orientation ( $\alpha$ angles distribution) and associated kappa values is shown in the bottom row. (h) Histograms of cone polarity scores in mice subjected to different light environments. LD: light:dark cycles, DD: dark:dark cycles, DDE12: dark-reared between E12.5 and sacrifice at P30, DDP9: dark-reared between P9 and sacrifice at P30, DDP12: dark-reared between P12 and sacrifice at P30, DDP16: dark-reared between P16 and sacrifice at P30, Late LD: darkreared between E12.5 and P22, then reared on a light-dark cycle until sacrifice at P360, Late DD: mice dark reared between P120 and sacrifice of P150, LD control: P30 mice reared in light-dark cycles. Graph shows mean \pm s.d. $n$ indicates biological replicates. Total number of cones analyzed: DDE12 $=3417$, DDP9 $=1663$, DDP12 $=1070$, DDP16 $=886$, Late LD $n=$ 1309 cones, Late DD $n=1077$, LD control $n=1224$ cones. One-way ANOVA $p<0.0001$. Dunnet's multi-comparison test ${ }^{* * *} p<0.001,{ }^{*} p<0.05$. (i) Violin plots of cone basal body eccentricity ( $n=3$ animals, 360 cells counted for each condition).

\section{Cone phototransduction is required to establish PCP}

The above results indicate that light is required to instruct cone PCP, but whether light acts directly in cones, or indirectly through other photosensitive cells of the retina was still unclear. To clarify the cellular origin of light-dependent polarization, we investigated cone PCP in mouse mutants of Gnat1 and Gnat2, which code for the transducin alpha subunits of rods (Gat1) and cones (Gat2), respectively. Importantly, while both rod and cone photosensitivity is abolished in Gnat1 $1^{\text {null/null }}$ and Gnat $2^{\text {null/null }}$ mouse lines, the general anatomy of the retina and the morphology of photoreceptors are unaffected $(23,24)$. We therefore studied cone centrosome orientation in Gnat1 ${ }^{\text {null/null. }}$; Gnat2 $2^{\text {null/null }}$ mice, in which both rods and cones have lost photosensitivity, and in Gnat1 ${ }^{\text {null/null; }}$ Gnat $2^{+/ n u l l}$ mice, in which only cones are photosensitive. We found that cone PCP is normal in Gnat1 ${ }^{\text {null/null. }}$ Gnat2 ${ }^{+/ \text {null }}$ mice $(\kappa=1.5 \pm 0.2$; Fig. $3 a, c)$, but disrupted in Gnat $1^{\text {null/null; }}$; Gnat2 ${ }^{\text {null/null }}$ mice $(\kappa=0.4 \pm 0.1$; Fig. 3b, c; Fig. S3a, b). Similar to light deprivation experiments, the loss of photosensitivity in rods and cones had no impact on the eccentricity of centrosomes in cones (Fig. 3d). These results indicate that cone phototransduction is necessary to mediate planar polarization and identify a non-canonical role for cone transducin in retina morphogenesis. 

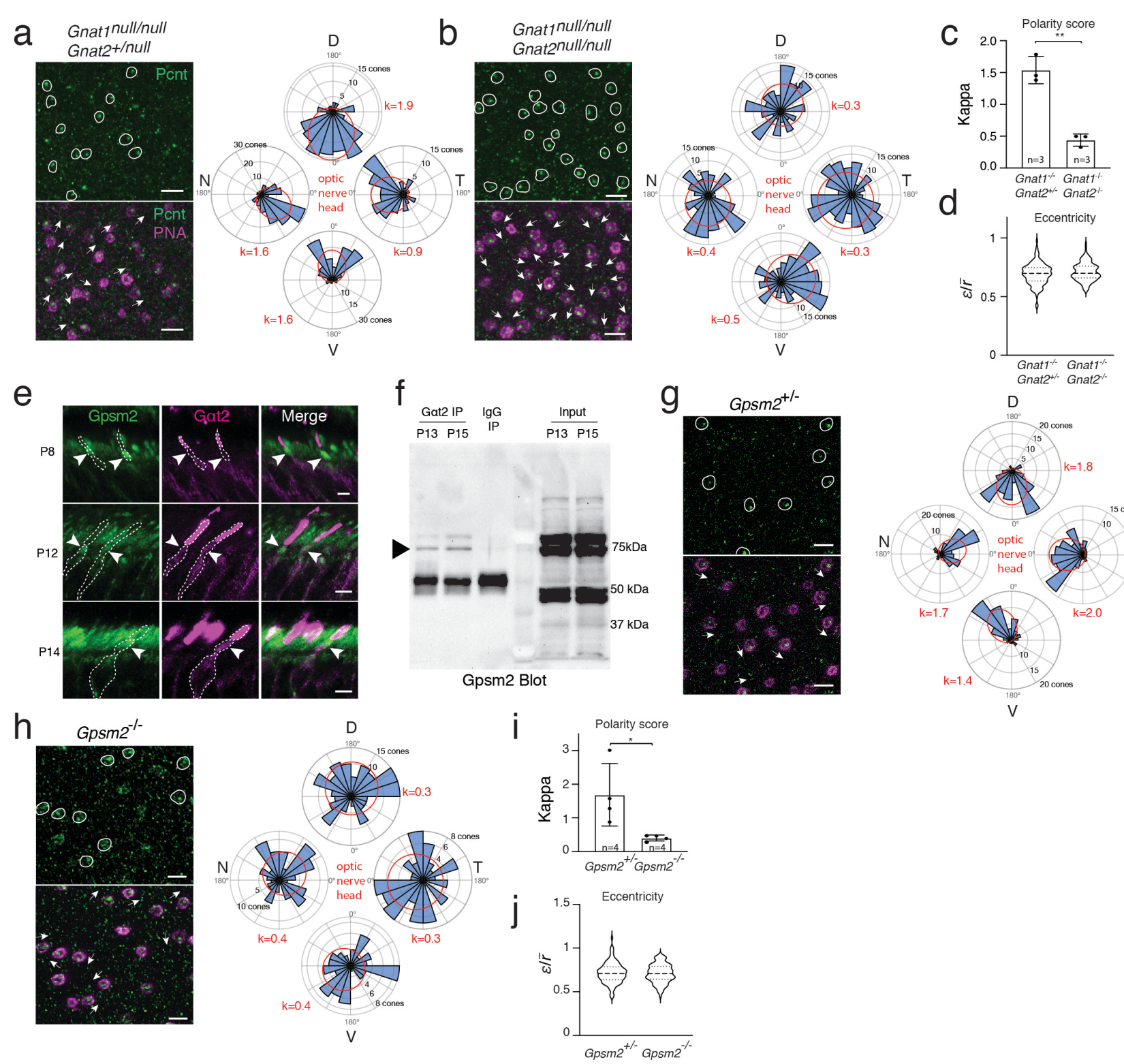

\section{Figure 3. Non-canonical pathway underlying cone PCP establishment}

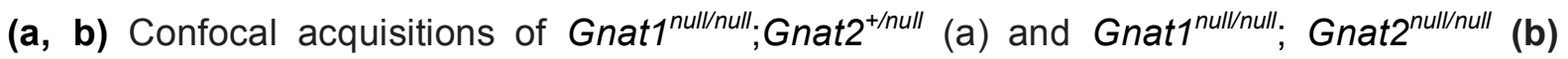
retina flat-mounts stained for pericentrin (Pcnt, green) and Peanut agglutinin (PNA, magenta). Representative examples of cones (outlined) with various centrosome orientation (arrows) are shown. For each genotype, a representative rose plot of centrosome orientation ( $\alpha$-angles distribution) and the associated kappa value is shown. (c) Histogram of cone

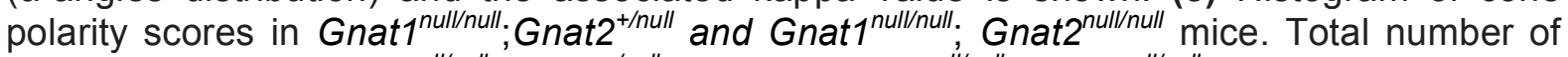
cones analyzed: Gnat1 ${ }^{\text {null/null }} ;$ Gnat2 $^{+/ \text {null }}=1649$, Gnat1 $^{\text {null/null }} ;$ Gnat2 $^{\text {null/null }}=1999$ cones. Twotailed unpaired t-test: $p=0.0013\left({ }^{* *} p<0.01\right)$. (d) Violin plots of cone centrosome eccentricity for each genotype ( $n=3$ animals, 360 cells counted for each genotype). (e) Confocal acquisitions of mouse retina sections stained for Gpsm2 (green) and Gat2 (magenta) at different developmental stages, as indicated. Arrowheads point to the cone IS and the dotted line delineate individual cones. (f) Co-immunoprecipitation (IP) of Gat2 and Gpsm2 from mouse retina extracts at $\mathrm{P} 13$ and $\mathrm{P} 15$. Rabbit $\lg \mathrm{g}(\mathrm{IgG})$ were used as IP controls at P13. $10 \%$ input form $\mathrm{P} 13$ and $\mathrm{P} 15$ whole retina protein extracts are shown. Gpsm2 specific band 
migrates at $\sim 75 \mathrm{kDa}$ (arrowhead, see also Figure. S4). (g, h) Confocal acquisitions of $\mathrm{Gpsm}^{+/-}(\mathrm{g})$ and $\mathrm{Gpsm}^{-/-}$(h) retina flat-mounts stained for pericentrin (Pcnt, green) and Peanut agglutinin (PNA, magenta). Representative examples of cones (outlined) with various centrosome orientation (arrows) are shown. For each genotype, a representative rose plot of centrosome orientation ( $\alpha$-angles distribution) and the associated kappa value is shown. (i) Histogram of cone polarity scores in $\mathrm{Gpsm}^{+/-}$and $\mathrm{Gpsm}^{-/-}$mice. Graph shows mean \pm s.d. $\mathrm{n}$ indicates biological replicates. Total number of cones analyzed: $\mathrm{Gpsm}^{+/-}=$ 1574 cones, Gpsm2 $2^{-\alpha} 1654$ cones. Dorsal (D); Nasal (N); Temporal (T); Ventral (V). Twotailed unpaired t-test $p=0.03\left({ }^{*} p<0.05\right)$. (j) Violin plots of cone centrosome eccentricity $(n=3$ animals, 360 cells counted for each genotype).

\section{Gpsm2 interacts with Gat2 and is required to establish cone PCP}

We next investigated how cone phototransduction could mediate repositioning of the centrosomes to establish PCP. Of note, cone transducin (Gat2) is encoded by the Gnat2 gene, which arose through duplication of an ancestral Gnai gene belonging to a family of heterotrimeric G-proteins, whose activity is modulated by Gpsm2 to regulate cell polarity in a wide variety of developmental contexts $(16,25,26)$. To investigate whether Gpsm2 might play a part in cone PCP, we first stained mouse retinas for Gat2 and Gpsm2 during the critical window of cone PCP establishment, using a Gpsm2 antibody that we validated on $\mathrm{Gpsm}^{-/-}$samples by western blot and immunostaining (Fig. S4). We found that Gat2 and Gpsm2 co-localize in the IS of cones between P8 and P14 (Fig. 3e). To ask whether both proteins form a complex, we immunoprecipitated Gat2 from whole retina protein extracts at P13 and P15 and immunoblotted for Gpsm2. We found that Gpsm2 interacts with Gat2 (Fig. 3f), suggesting that they might function together to instruct cone PCP. To investigate this possibility, we studied cone centrosome orientation in mouse knockouts of Gpsm2. While cone PCP is clearly established in $\mathrm{Gpsm}^{+/-}$mice $(\mathrm{k}=1.7 \pm 0.9$; Fig. $3 \mathrm{~g}$, i; Fig. S3c, d), it is disrupted in Gpsm2 $2^{-/-}$mice ( $k=0.4 \pm 0.1$; Fig. 3h, i; Fig. S3c, d). This finding was further confirmed using the FIBSEM analysis (Fig. S5). In contrast to previous findings in cochlear hair cells, however, the loss of cone PCP in Gpsm2 $2^{-/}$is not accompanied by a disruption of the intrinsic polarity, as eccentric localization of cone centrosomes is not affected (Fig. 3j).

Together, these results identify a molecular pathway downstream of light absorption that underlies cone PCP establishment in the mouse retina.

\section{Cone PCP contributes to spatio-temporal visual discrimination in mice}

We then asked whether cone PCP might play a part in visual perception. To address this question, we studied mice with disrupted cone PCP. Since dark-rearing impairs the maturation of interneurons of the retina (27-29) and cones do not respond to light stimulation in Gnat2 ${ }^{\text {null/null }}$ mice, we thought that $\mathrm{Gpsm} 2^{-/-}$mice were the most appropriate model for these experiments. As previously shown, we found that 
Gpsm2 $^{-/-}$mice exhibit normal retinal lamination and present no signs of retinal degeneration (Fig. S6a) (30). In addition, the histological observation of $\mathrm{Gpsm} 2^{-/-}$ cones showed that they are indistinguishable from those of $G p s m 2^{+/-}$in crosssections (Figure S6b), suggesting that cone PCP is the only morphological feature disrupted in adult $\mathrm{Gpsm}^{-/-}$retinas.

We first tested whether cone PCP might be important to coordinate global cone activity in the retina. We recorded flash electroretinograms in $\mathrm{Gpsm}^{-/-}$mice and Gpsm2 $^{+/}$control mice in scotopic and photopic conditions to test rod and cone responses, respectively. In both conditions, the electroretinogram waveforms and time courses were not significantly different between $\mathrm{Gpsm}^{-/}$and $\mathrm{Gpsm}^{+/-}$mice (Fig. S6c-f), suggesting that cone PCP does not contribute to the global response of cone photoreceptors.

Next, we examined whether cone PCP might contribute to fine-detail visual discrimination. To test this hypothesis, we measured the optomotor head-tracking responses to a rotatory grating of different spatial frequencies under photopic conditions in $\mathrm{Gpsm}^{+/+}, \mathrm{Gpsm}^{+/-}$, and $\mathrm{Gpsm} 2^{-/-}$mice (Fig. 4a). We found that adult $\mathrm{Gpsm}^{-/-}$mice exhibit a significant decrease in spatial frequency threshold compared to $\mathrm{Gpsm}^{+/+}$and $\mathrm{Gpsm} 2^{+/-}$mice (Fig 4b). Importantly, Gpsm2 $2^{-/-}$mice perform similarly to control mice prior to the establishment of PCP. At P17, however, when cone PCP is in place in controls (see Fig. 2a-c), a significant difference in test performance is detected between controls and $\mathrm{Gpsm} 2^{-/-}$mice. This difference further increases at P23 and then reaches a plateau (Fig. 4c). These results indicate that cone PCP is necessary for normal visual acuity in mice.
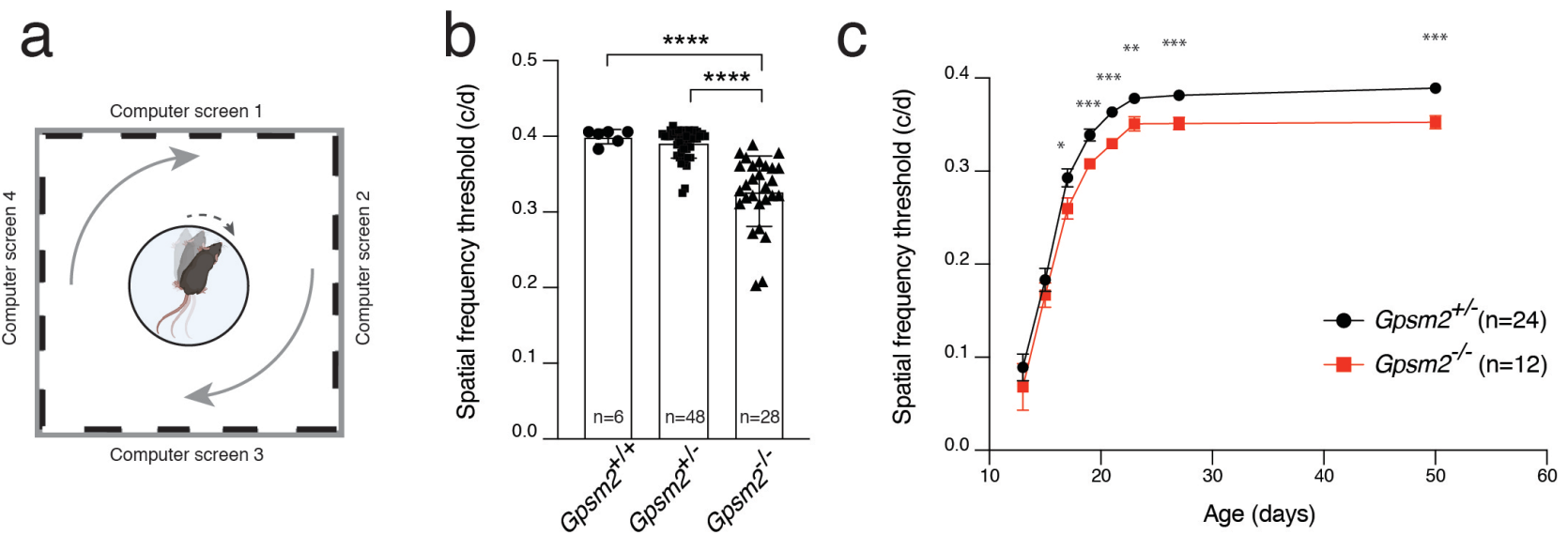

Figure 4. Reduced spatial frequency detection in mice with altered cone PCP

(a) Schematic representation of the optomotor head-tracking assay. Animals are placed in a box surrounded by a rotating drum with gratings of different spatial frequencies. The maximum spatial frequency eliciting a visual response, which is recorded by reflex movements of the head, is compared between mice of different genotypes. (b) Histogram representing the mean \pm s.d. of spatial frequency threshold (cycles per degree) for eyes 
bioRxiv preprint doi: https://doi.org/10.1101/2021.04.21.440771; this version posted April 21, 2021. The copyright holder for this preprint (which was not certified by peer review) is the author/funder. All rights reserved. No reuse allowed without permission.

from each indicated mouse genotype. Each data point represents a different eye. One way ANOVA $p<0.0001$ and a posteriori multi comparison t-test with Tukey corrections (c) Spatialfrequency threshold measurements as a function of time (P13, P15, P17, P19, P21, P23, P27, P50). Each data point represents the mean \pm s.e.m. Two-way ANOVA $p<0.0001$ for age factor and $p<0.0001$ for genotype factor and a posteriori multi comparison t-test ${ }^{*} p<0.05$, ${ }^{* *} p<0.01,{ }^{* * *} p<0.001$. 


\section{DISCUSSION}

Planar polarization of basal bodies is a feature shared across a wide diversity of epithelial cells $(2,31,32)$. Photoreceptors share many features with cochlear hair cells, as both exhibit an eccentric primary cilium. However, while the apical surface of hair cells displays easily identified v-shaped stereocilia that all point in the same direction, our FIBSEM imaging of photoreceptors has not revealed any other polarized feature than the connecting cilium, making cone PCP inconspicuous. This might account for the century delay between the discovery of PCP in the ear (33), and here, in the retina.

By sampling critical information in the environment, sensory organs are particularly prone to morphological changes related to their function. While it remains to be determined whether cone PCP in mammals is an adaptation to nocturnal vision, it is worth noting that cone PCP was reported in the zebrafish retina, a diurnal animal (34). In zebrafish, red-, green-, and blue-cones harbor an eccentric basal body pointing towards the optic nerve head, similar to what we report in mice, while UVcone and rod PRs do not. Although the mechanisms and role of cone PCP were not identified in fish, it was reported to arise at late stages of development, suggesting that phototropism, similar to what we find in mice, may be involved. In mammals, potential planar polarity was reported in the diurnal cone-dominated retina of the tree shrew (35), but a more thorough characterization awaits further studies. The primate retina is also of particular interest, as it contains a cone-rich macula at the center, surrounded by a rod-dominated periphery. It will be important to study PCP in detail in these animals to help determine whether cone PCP is the rule, or an exception, across mammals.

Our finding that rods do not exhibit PCP prompted us to explore a potential role for cone PCP in visual acuity, a function predominantly supported by cones. We show that disruption of cone PCP decreases the spatio-temporal detection threshold, uncovering a role for planar cell organization in vision and suggesting that PCP may qualitatively optimize spatio-temporal acquisition of information. Psychophysical experiments conducted in humans reported that the retina is more sensitive to light entering the center of the pupil than light passing through its periphery and thereby hitting photoreceptors at an angle, a phenomenon referred to as the Stiles-Crawford effect $(36,37)$. While this effect is prominent in cones, it is weak in rods $(38)$, and optical properties of individual rod and cone PRs have failed to identify a convincing cellular origin of this phenomenon in humans $(39,40)$. Interestingly, a recent study in mice reported that the rectilinear orientation of IS and OS of rod photoreceptors, which is instructed by light between P0 and P8, is involved in the Stiles-Crawford effect (41). As rods are thought to play a minor part in this effect, it is likely that conespecific mechanisms are also contributing. Ex-vivo experiments suggest that, in contrast to rods, cones do not exhibit a dynamic phototropic realignment of their IS 
and $\mathrm{OS}$ in adult mice $(42,43)$, suggesting alternative mechanisms. The potential role of cone PCP in the Stiles-Crawford effect remains unknown, but we propose that the coordinated orientation of cilia may physically restrict cones to exclusively detect light that is paraxial to the eye optics, filtering out intraocular stray light that deviates from this axis. Our results showing that cone PCP cannot be reversed by rearing adult mice in the dark is in agreement with psychophysical observations showing that monocular light deprivation fails to alter the Stiles-Crawford effect in adult human subjects (44). Should cone PCP be conserved in humans, it may be possible to test its role in the Stiles-Crawford effect by performing psychophysical experiments on patients bearing a mutation in the GPSM2 gene, which cause the ChudleyMcCullough syndrome, a congenital disorder characterized by hearing loss and various brain anomalies $(45,46)$. Based on our work in mice, we expect that these patients would have disrupted cone PCP and perform poorly in tasks testing the Stiles-Crawford effect.

Molecularly, our data indicate that Gpsm2 and Gat2 interact and are both required to establish cone PCP. In asymmetric cell division, Gpsm2 functions as an adaptor protein linking Gai subunits anchored at the apical cell cortex to the microtubule- and dynein-associated protein Numa, leading to capture of aster microtubules and reorientation of the mitotic spindle. In a striking example of pleiotropy, we found that the position of the microtubule-based basal bodies in cones is also regulated by Gpsm2 and the Gai-related protein cone transducin (Gat2). While Gpsm2 and Gat2 may constitute the intracellular levers to position the basal body in cones, the mechanisms coordinating basal body positions across the retina remain to be determined. The unique cellular architecture of photoreceptors makes it unlikely, although not impossible, that canonical PCP pathways coordinate basal body positions across the tissue. Indeed, photoreceptor cell junctions, through which PCP signaling normally propagates, are located at the base of the IS, whereas the basal bodies are distantly located at the tip. Accordingly, knockdown of the core PCP gene Vangl2 in cones does not disrupt cone PCP in the zebrafish retina, suggesting that it may also rely on a non-canonical pathway (47).

Asymmetric activation of G-protein coupled receptor (GPCR) by an extracellular ligand is generally involved in setting-up PCP in various epithelia. As light is the "ligand" sensed by the photoreceptor-specific GPCR Opsin, our observations raise the possibility that a gradient of light, rather than molecules, might instruct basal body positioning during cone maturation. In such a model, Gat2 may constitute the molecular link between light and Gpsm2, forming a non-canonical pathway to instruct the spatial localization of cone basal bodies across the retina. But if a gradient of light mediates cone PCP, what could be the source of this gradient? We envision at least two possibilities: a global gradient of light over the scale of the eye, where the central retina might receive more light than the periphery, or a local gradient at the level of individual cones. As Müller glial cells are known to funnel light directly from the vitreous to the photoreceptors (48-50), they might play a part in establishing a 
local gradient of light. The 1:1 ratio of cone-to-Müller cell reported in various vertebrate retinas is consistent with this possibility (51). Further work will be required to determine whether a light gradient is involved and how it is established.

In conclusion, our results identify a non-canonical pathway of PCP mediated by light, cone transducin, and Gpsm2. As several other regions of the developing brain demonstrate photosensitivity $(52,53)$, this work raises the exciting possibility that light may generally regulate tissue morphogenesis in the CNS. 


\section{ACKNOWLEDGMENTS}

We thank Jessica Barthe, Marie-Claude Lavalée and Caroline Dubé for help with the animal colony, Simone Terouz for histology, and François Couderc for genotyping. We are grateful to Samer Hattar (Johns Hopkins) for sharing the Gnat1/Gnat2 mutant mice, K. Sears, J. Mui, L. Monaghan, L. Mongeon and W. Leelapornpisit at the Facility for Electron Microscopy Research for help in sample processing and data collection by FIBSEM and the Vision Health Research Network (VHRN) for tissues. This work was funded by grants from the Canadian Institutes of Health Research (FDN-159936 to M.C.; 130376 to C.M.), The Brain Canada/Weston Foundation, and the Natural Sciences and Engineering Research Council (to C. C.). M.H. was funded by a postdoctoral fellowship from the Fondation pour la recherche médicale française (FRM). M.C. is a FRQS Emeritus Research Scholar and holds the IRCM Foundation Gaëtane and Roland Pillenière Chair in Retina biology. 


\section{REFERENCES}

1. R. S. Molday, O. L. Moritz, Photoreceptors at a glance. J Cell Sci. 128, 40394045 (2015).

2. D. Devenport, The cell biology of planar cell polarity. J Cell Biol. 207, 171-179 (2014).

3. D. F. Ready, T. E. Hanson, S. Benzer, Development of the Drosophila retina, a neurocrystalline lattice. Developmental Biology. 53, 217-240 (1976).

4. M. Simons, M. Mlodzik, Planar cell polarity signaling: from fly development to human disease. Annu Rev Genet. 42, 517-540 (2008).

5. T. R. Clandinin, S. L. Zipursky, Making connections in the fly visual system. Neuron. 35, 827-841 (2002).

6. M. Langen, E. Agi, D. J. Altschuler, L. F. Wu, S. J. Altschuler, P. R. Hiesinger, The Developmental Rules of Neural Superposition in Drosophila. Cell. 162, 120-133 (2015).

7. C.-H. Sung, J.-Z. Chuang, The cell biology of vision. The Journal of Cell Biology. 190, 953-963 (2010).

8. T. G. Wensel, Signal transducing membrane complexes of photoreceptor outer segments. Vision Research. 48, 2052-2061 (2008).

9. M. T. Butler, J. B. Wallingford, Planar cell polarity in development and disease. Nat Rev Mol Cell Biol. 18, 375-388 (2017).

10. M. Montcouquiol, M. W. Kelley, Development and Patterning of the Cochlea: From Convergent Extension to Planar Polarity. Cold Spring Harb Perspect Med. 10 (2020), doi:10.1101/cshperspect.a033266.

11. J. Ezan, L. Lasvaux, A. Gezer, A. Novakovic, H. May-Simera, E. Belotti, A.-C. Lhoumeau, L. Birnbaumer, S. Beer-Hammer, J.-P. Borg, A. Le Bivic, B. Nürnberg, N. Sans, M. Montcouquiol, Primary cilium migration depends on Gprotein signalling control of subapical cytoskeleton. Nat Cell Biol. 15, 11071115 (2013).

12. B. Tarchini, C. Jolicoeur, M. Cayouette, A molecular blueprint at the apical surface establishes planar asymmetry in cochlear hair cells. Dev. Cell. 27, 88102 (2013).

13. Q. Du, P. T. Stukenberg, I. G. Macara, A mammalian Partner of inscuteable binds NuMA and regulates mitotic spindle organization. Nature Cell Biology. 3, 1069-1075 (2001).

14. J. A. Knoblich, Mechanisms of asymmetric cell division during animal development. Current Opinion in Cell Biology. 9, 833-841 (1997). 
15. L. Pirovano, S. Culurgioni, M. Carminati, A. Alfieri, S. Monzani, V. Cecatiello, C. Gaddoni, F. Rizzelli, J. Foadi, S. Pasqualato, M. Mapelli, Hexameric NuMA:LGN structures promote multivalent interactions required for planar epithelial divisions. Nat Commun. 10 (2019), doi:10.1038/s41467-019-09999-w.

16. A. Shitamukai, F. Matsuzaki, Control of asymmetric cell division of mammalian neural progenitors. Dev Growth Differ. 54, 277-286 (2012).

17. P. D. Calvert, K. J. Strissel, W. E. Schiesser, E. N. Pugh, V. Y. Arshavsky, Light-driven translocation of signaling proteins in vertebrate photoreceptors. Trends in Cell Biology. 16, 560-568 (2006).

18. T. D. Lamb, D. M. Hunt, Evolution of the vertebrate phototransduction cascade activation steps. Developmental Biology. 431, 77-92 (2017).

19. V. S. Kerov, M. Natochin, N. O. Artemyev, Interaction of transducin-alpha with LGN, a G-protein modulator expressed in photoreceptor cells. Mol. Cell. Neurosci. 28, 485-495 (2005).

20. K. S. Nair, A. Mendez, J. B. Blumer, D. H. Rosenzweig, V. Z. Slepak, The presence of a Leu-Gly-Asn repeat-enriched protein (LGN), a putative binding partner of transducin, in ROD photoreceptors. Invest. Ophthalmol. Vis. Sci. 46, 383-389 (2005).

21. L. Landler, G. D. Ruxton, E. P. Malkemper, Circular data in biology: advice for effectively implementing statistical procedures. Behav Ecol Sociobiol. 72 (2018), doi:10.1007/s00265-018-2538-y.

22. J. M. Daum, Ö. Keles, S. J. Holwerda, H. Kohler, F. M. Rijli, M. Stadler, B. Roska, The formation of the light-sensing compartment of cone photoreceptors coincides with a transcriptional switch. eLife. 6, doi:10.7554/eLife.31437.

23. J. J. Alexander, Y. Umino, D. Everhart, B. Chang, S. H. Min, Q. Li, A. M. Timmers, N. L. Hawes, J.-J. Pang, R. B. Barlow, W. W. Hauswirth, Restoration of cone vision in a mouse model of achromatopsia. Nat Med. 13, 685-687 (2007).

24. M. Miyamoto, M. Aoki, K. Hirai, S. Sugimoto, K. Kawasaki, R. Imai, A nonsense mutation in Gnat1, encoding the a subunit of rod transducin, in spontaneous mouse models of retinal dysfunction. Experimental Eye Research. 90, 63-69 (2010).

25. A. H. Hansen, C. Duellberg, C. Mieck, M. Loose, S. Hippenmeyer, Cell Polarity in Cerebral Cortex Development-Cellular Architecture Shaped by Biochemical Networks. Front Cell Neurosci. 11 (2017), doi:10.3389/fncel.2017.00176.

26. J. A. Knoblich, Asymmetric cell division: recent developments and their implications for tumour biology. Nat. Rev. Mol. Cell Biol. 11, 849-860 (2010).

27. F. A. Dunn, L. Della Santina, E. D. Parker, R. O. L. Wong, Sensory Experience Shapes the Development of the Visual System's First Synapse. Neuron. 80, 1159-1166 (2013). 
28. A. Giovannelli, S. Di Marco, R. Maccarone, S. Bisti, Long-term dark rearing induces permanent reorganization in retinal circuitry. Biochem. Biophys. Res. Commun. 365, 349-354 (2008).

29. A. Tiriac, B. E. Smith, M. B. Feller, Light prior to eye-opening promotes retinal waves and eye-specific segregation. Neuron. 100, 1059-1065.e4 (2018).

30. M. Lacomme, B. Tarchini, C. Boudreau-Pinsonneault, C. Monat, M. Cayouette, The LGN protein promotes planar proliferative divisions in the neocortex but apicobasal asymmetric terminal divisions in the retina. Development. 143, 575581 (2016).

31. R. Bayly, J. D. Axelrod, Pointing in the right direction: new developments in the field of planar cell polarity. Nat Rev Genet. 12, 385-391 (2011).

32. L. V. Goodrich, D. Strutt, Principles of planar polarity in animal development. Development. 138, 1877-1892 (2011).

33. A. Corti, Royal College of Surgeons of England, Recherches sur l'organe de l'ouïe des mammifères. 1. ptie. Limaçon ([Leipzig: s.n.], 1851; http://archive.org/details/b2234410x).

34. M. Ramsey, B. D. Perkins, Basal bodies exhibit polarized positioning in zebrafish cone photoreceptors. J. Comp. Neurol. 521, 1803-1816 (2013).

35. W. Knabe, H. Kuhn, Disk formation in retinal cones of Tupaia belangeri (Scandentia). Cell Tissue Res. 292, 67-76 (1998).

36. W. S. Stiles, P. D, B. S. B. H. Crawford, The luminous efficiency of rays entering the eye pupil at different points. Proc. R. Soc. Lond. B. 112, 428-450 (1933).

37. W. S. Stiles, B. H. Crawford, J. H. Parsons, The effect of a glaring light source on extrafoveal vision. Proceedings of the Royal Society of London. Series B Biological Sciences. 122, 255-280 (1937).

38. F. Flamant, W. S. Stiles, The directional and spectral sensitivities of the retinal rods to adapting fields of different wave-lengths. J. Physiol. (Lond.). 107, 187202 (1948).

39. J. M. Enoch, The relationship between retinal receptor orientation and photoreceptor optics. Int Ophthalmol Clin. 18, 41-80 (1978).

40. G. Westheimer, Directional sensitivity of the retina: 75 years of Stiles-Crawford effect. Proceedings of the Royal Society of London B: Biological Sciences. 275, 2777-2786 (2008).

41. Z. Chai, D. Silverman, G. Li, D. Williams, E. Raviola, K.-W. Yau, Lightdependent photoreceptor orientation in mouse retina. Sci Adv. 6 (2020), doi:10.1126/sciadv.abe2782. 
42. R. Lu, A. M. Levy, Q. Zhang, S. J. Pittler, X. Yao, Dynamic near-infrared imaging reveals transient phototropic change in retinal rod photoreceptors. $J$ Biomed Opt. 18 (2013), doi:10.1117/1.JBO.18.10.106013.

43. B. Wang, Q. Zhang, R. Lu, Y. Zhi, X. Yao, Functional optical coherence tomography reveals transient phototropic change of photoreceptor outer segments. Opt. Lett., OL. 39, 6923-6926 (2014).

44. J. M. Enoch, R. D. Hamer, V. Lakshminarayanan, T. Yasuma, D. G. Birch, S. Yamade, Effect of monocular light exclusion on the Stiles-Crawford function. Vision Res. 27, 507-510 (1987).

45. A. R. Hamzeh, P. Nair, M. Mohamed, F. Saif, N. Tawfiq, M. T. Al-Ali, F. Bastaki, A novel nonsense GPSM2 mutation in a Yemeni family underlying ChudleyMcCullough syndrome. European Journal of Medical Genetics. 59, 337-341 (2016).

46. S. A. Mauriac, Y. E. Hien, J. E. Bird, S. D.-S. Carvalho, R. Peyroutou, S. C. Lee, M. M. Moreau, J.-M. Blanc, A. Gezer, C. Medina, O. Thoumine, S. BeerHammer, T. B. Friedman, L. Rüttiger, A. Forge, B. Nürnberg, N. Sans, M. Montcouquiol, Defective Gpsm2/Gai3 signalling disrupts stereocilia development and growth cone actin dynamics in Chudley-McCullough syndrome. Nat Commun. 8 (2017), doi:10.1038/ncomms14907.

47. P. Song, L. Dudinsky, J. Fogerty, R. Gaivin, B. D. Perkins, Arl13b Interacts With Vangl2 to Regulate Cilia and Photoreceptor Outer Segment Length in Zebrafish. Invest. Ophthalmol. Vis. Sci. 57, 4517-4526 (2016).

48. S. Agte, S. Junek, S. Matthias, E. Ulbricht, I. Erdmann, A. Wurm, D. Schild, J. A. Käs, A. Reichenbach, Müller Glial Cell-Provided Cellular Light Guidance through the Vital Guinea-Pig Retina. Biophys J. 101, 2611-2619 (2011).

49. K. Franze, J. Grosche, S. N. Skatchkov, S. Schinkinger, C. Foja, D. Schild, O. Uckermann, K. Travis, A. Reichenbach, J. Guck, Müller cells are living optical fibers in the vertebrate retina. Proc Natl Acad Sci U S A. 104, 8287-8292 (2007).

50. A. M. Labin, S. K. Safuri, E. N. Ribak, I. Perlman, Müller cells separate between wavelengths to improve day vision with minimal effect upon night vision. Nature Communications. 5 (2014), doi:10.1038/ncomms5319.

51. W. Lindenau, H. Kuhrt, E. Ulbricht, K. Körner, A. Bringmann, A. Reichenbach, Cone-to-Müller cell ratio in the mammalian retina: A survey of seven mammals with different lifestyle. Experimental Eye Research (2019), doi:10.1016/j.exer.2019.01.012.

52. D. Kojima, Y. Fukada, Non-visual photoreception by a variety of vertebrate opsins. Novartis Found Symp. 224, 265-279; discussion 279-282 (1999).

53. G. J. Schwartz, Light-activated neurons deep in the brain control body heat. Nature. 585, 351-352 (2020). 
bioRxiv preprint doi: https://doi.org/10.1101/2021.04.21.440771; this version posted April 21, 2021. The copyright holder for this preprint (which was not certified by peer review) is the author/funder. All rights reserved. No reuse allowed without permission. 